\title{
DERIVATIONS AND THE TRACE-CLASS OPERATORS
}

\author{
R. E. WEBER
}

\begin{abstract}
Let $\Re\left(\Delta_{A}\right)$ represent the range of the derivation generated by $A \in \mathscr{B}(\mathcal{H})$. It is shown that for each $n>2, \operatorname{tr}\left(T^{n}\right)=0$ for any trace-class operator $T \in\{A\}^{\prime}$ which is either (a) the weak limit of a sequence in $R\left(\Delta_{A}\right)$ or (b) a finite rank operator in the weak closure of $\mathscr{R}\left(\Delta_{A}\right)$. From this it follows that if $K \in\{A\}^{\prime}$ is a compact operator in the weak closure of $\Re\left(\Delta_{A}\right)$, then $K$ is quasinilpotent.
\end{abstract}

1. Introduction. Let $\mathcal{H}$ be a separable Hilbert space and $\Delta_{A}$ the derivation generated by $A \in \mathscr{B}(\mathcal{H})$ (the bounded linear operator on $\mathscr{B}(\mathcal{H})$ defined by $\Delta_{A}(X)=A X-X A$ for all $X \in \mathscr{B}(\mathcal{H})$ ). Kleinecke [6] has shown that $\{A\}^{\prime}$ $\cap \Re\left(\Delta_{A}\right)$ consists only of quasinilpotents and H. W. Kim [5] extended this to compact operators which are in both $\{A\}^{\prime}$ and the weak sequential closure of $\Re\left(\Delta_{A}\right)$. We will extend this result to show that compact operators in both $\{A\}^{\prime}$ and the weak closure of $\Re\left(\Delta_{A}\right)$ are quasinilpotent. Also necessary and sufficient conditions are given in order for the weak closure of the range of one derivation to be contained in another.

2. Notation. Let $\mathcal{T}$ be the ideal of trace-class operators, that is, all compact operators $T \in \mathscr{B}(\mathcal{F})$ for which the eigenvalues of $(T * T)^{1 / 2}$ (counted according to multiplicity) are summable. The ideal $\mathcal{T}$ admits a complex valued function $\operatorname{tr}(T)$ which has the characteristic properties of the trace for matrices. The trace function is defined by $\operatorname{tr}(T)=\Sigma_{n}\left(T e_{n}, e_{n}\right)$ where $\left\{e_{n}\right\}$ is any complete orthonormal sequence in $\mathcal{H}$. As Banach spaces, $\mathcal{T}$ may be identified with the conjugate space of the ideal $\mathcal{K}$ of compact operators by means of the linear isometry $T \rightarrow f_{T}$ where $f_{T}(X)=\operatorname{tr}(X T)$. Moreover, $\mathscr{B}(\mathcal{H})$ is the conjugate space of $\mathcal{T}$. The weak* (or ultraweakly) continuous linear functionals on $\mathscr{B}(\mathcal{H C})$ are those of the form $f_{T}$ for some $T \in \mathcal{T}$. Furthermore, the weak (or weak operator topology) continuous linear functionals on $\mathscr{B}(\mathcal{H})$ are those of the form $f_{T}$ where $T$ is of finite rank. (For a discussion of the above remarks see Dixmier [1].)

In the following, $R\left(\Delta_{A}\right)^{-}$will denote the weak closure and $\Re\left(\Delta_{A}\right)^{-}$will denote the weak* closure of the range of derivation generated by $A \in \mathscr{B}(\mathcal{H})$.

3. We begin with the following result on the trace of certain operators.

THEOREM 1. Let $A \in \mathscr{B}(\mathcal{H})$ and let $T$ be a trace-class operator which

Received by the editors March 24, 1978.

AMS (MOS) subject classifications (1970). Primary 47B47; Secondary 47B05.

Key words and phrases. Derivation ranges, trace-class, compact operator, quasinilpotent operator. 
commutes with $A$. If either

(a) $T$ is the weak limit of a sequence of elements in $\Re\left(\Delta_{A}\right)$ or

(b) $T$ is of finite rank and in the weak closure of $R\left(\Delta_{A}\right)$ then $\operatorname{tr}\left(T^{n}\right)=0$ for all $n \geqslant 2$.

Proof. If the trace-class operator $T$ commutes with $A$, then

$$
\begin{aligned}
f_{T}(A X-X A) & =\operatorname{tr}(T(A X-X A))=\operatorname{tr}(T A X-T X A) \\
& =\operatorname{tr}(T A X-T A X)=0 .
\end{aligned}
$$

Hence $f_{T}$ is a weak* continuous linear functional which vanishes on $\mathcal{R}\left(\Delta_{A}\right)$. Now if $\left\{A X_{n}-X_{n} A\right\}$ converges weakly to $T$ then $\left\|A X_{n}-X_{n} A\right\|$ is bounded (see [2, Problem 40]) and since the weak and weak* topologies are equivalent on bounded sets,

$$
f_{T}(T)=\operatorname{tr}\left(T^{2}\right)=0 .
$$

If $T$ is of finite rank and commutes $A$, then $f_{T}$ is a weakly continuous linear function which vanishes on $\Re\left(\Delta_{A}\right)$. Hence if $T$ is also in the weak closure of $\Re\left(\Delta_{A}\right)$ then

$$
f_{T}(T)=\operatorname{tr}\left(T^{2}\right)=0 .
$$

In either case, since $T^{n} \Re\left(\Delta_{A}\right) \subset \Re\left(\Delta_{A}\right)$,

$$
\operatorname{tr}\left(T^{n}\right)=\operatorname{tr}\left(T T^{n-1}\right)=f_{T}\left(T^{n-1}\right)=0
$$

for all $n \geqslant 2$.

The set of Hilbert-Schmidt operators $(\mathcal{S})$ on $\mathcal{H}$ equipped with the trace inner product $(A, B)=\operatorname{tr}\left(A B^{*}\right)$ is a Hilbert space which contains $\mathcal{T}$ [7, p. 34]. In the following theorem $R\left(\Delta_{A}\right)^{\sim}$ will denote the Hilbert-Schmidt closure of the range of a derivation generated by a Hilbert-Schmidt operator $A$.

Theorem 2. If $A \in \mathcal{S}$ and $T \in\{A\}^{\prime} \cap \mathcal{R}\left(\Delta_{A}\right)^{\sim}$ then $\operatorname{tr}\left(T^{n}\right)=0$ for all $n \geqslant 2$.

Proof. The restriction of $\Delta_{A}$ to $\delta$ is a bounded linear operator on $\delta$ with adjoint equal to $\Delta_{A^{*}}$ restricted to $\delta$. Hence $\delta=\Re\left(\Delta_{A} \mid \mathcal{\delta}\right)^{\sim} \bigoplus\left\{A^{*}\right\}^{\prime} \cap \mathcal{\delta}$. Also, $R\left(\Delta_{A} \mid \delta\right)^{\sim}=\Re\left(\Delta_{A}\right)^{\sim}$ (see Theorem 2 of [8]). Therefore,

$$
\operatorname{tr}\left(T^{2}\right)=\left(T, T^{*}\right)=0 .
$$

For $n>2, T^{n-1} \in\{A\}^{\prime} \cap \mathcal{R}\left(\Delta_{A}\right) \sim$. Hence

$$
\operatorname{tr}\left(T^{n}\right)=\left(T^{n-1}, T^{*}\right)=0 .
$$

Operators satisfying the above theorems have a distinct spectral property which follows from the following lemma (the proof of which was suggested by Y. Ho).

LEMMA. If $T$ is a trace-class operator with $\operatorname{tr}\left(T^{n}\right)=0$ for all $n \geqslant 1$ then $\sigma(T)=0$.

Proof. Assume $\sigma(T) \neq\{0\}$ and let $\lambda \neq 0$ be an isolated eigenvalue of 
finite multiplicity. Also, let $\gamma$ be a Jordan curve such that $\sigma(T)$ intersects the interior of $\gamma$ only at $\lambda$. Then

$$
E=\frac{1}{2 \pi i} \int_{\gamma}(z-T)^{-1} d z
$$

is the Riesz projection determined by $\gamma$. Hence $\lambda \in \sigma(E T)$ and $E \in\{T\}^{\prime \prime}$. By Runge's Theorem, there exists a sequence of polynomials $\left\{\boldsymbol{P}_{m}\right\}$ such that

$$
\lim \left\|P_{m}(T)-E\right\|=0 .
$$

If $\operatorname{tr}\left(T^{n}\right)=0$ for all $n \geqslant 1$, then

$$
\operatorname{tr}\left((E T)^{n}\right)=\operatorname{tr}\left(E T^{n}\right)=\lim _{m \rightarrow \infty} \operatorname{tr}\left(P_{m}(T) T^{n}\right)=0
$$

for all $n \geqslant 1$. Since $E(\mathcal{H})$ is finite dimensional, Jacobson's Lemma (see [3, p. 273] for example) implies ET is nilpotent which contradicts the assumption that $\lambda \neq 0$.

COROLlaRY. If $T$ satisfied either Theorem 1 or Theorem 2, then $\sigma(T)=0$.

Proof. If $T$ satisfies either of the theorems, then $\operatorname{tr}\left(T^{2 n}\right)=0$ for all $n \geqslant 1$. It follows from the lemma that $\sigma\left(T^{2}\right)=0$ and hence $\sigma(T)=0$.

We are now ready to prove the following theorem.

TheOREM 3. Let $A \in \Re(\mathcal{H})$. If $K$ is a compact operator in $\{A\}^{\prime} \cap \Re\left(\Delta_{A}\right)^{-}$, then $K$ is quasinilpotent.

Proof. Assume $\lambda \in \sigma(K)$ with $\lambda \neq 0$ an isolated eigenvalue of finite multiplicity. Let $E$ be the Riesz projection as defined in the proof of the lemma. Since $E \in\{K\}^{\prime \prime}$, then $E K \in\{A\}^{\prime}$ and $E \Re\left(\Delta_{A}\right) \subset \Re\left(\Delta_{A}\right)$. Multiplication is weakly continuous with respect to one argument [2, Problem 92]. Hence $E K \in \mathscr{R}\left(\Delta_{A}\right)^{-}$, and since $E K$ has finite rank, it satisfies part (b) of Theorem 1. By the corollary, $\sigma(E K)=0$ which contradicts the assumption that $\lambda \neq 0$.

4. The above leads us to consider the relationship between the closures of two derivation ranges. In the following theorem we let $\mathcal{F}$ denote the ideal of finite rank operators.

Theorem 4. Let $A, B \in \mathscr{B}(\mathcal{H})$. Then

(a) $\mathscr{R}\left(\Delta_{B}\right)^{-} \subset \mathcal{R}\left(\Delta_{A}\right)^{-}$if and only if $\{A\}^{\prime} \cap \mathcal{F} \subset\{B\}^{\prime} \cap \mathcal{F}$ and

(b) $\Re\left(\Delta_{B}\right)=\mathcal{R}\left(\Delta_{A}\right)=$ if and only if $\{A\}^{\prime} \cap \mathcal{T} \subset\{B\}^{\prime} \cap \mathcal{T}$.

Proof. To prove (a) assume $\{A\}^{\prime} \cap \mathcal{F} \subset\{B\}^{\prime} \cap \mathcal{F}$ and let $f_{T}$ be a weakly continuous linear functional which annihilates $\Re\left(\Delta_{A}\right)$, then

$$
0=f_{T}(A X-X A)=\operatorname{tr}(T(A X-X A))=\operatorname{tr}((T A-A T) X)
$$

for all $X \in \mathscr{B}(\mathcal{H})$, hence $T A=A T$ and $T \in\{A\}^{\prime} \cap \mathscr{F} \subset\{B\}^{\prime} \cap \mathscr{F}$. Therefore, 


$$
f_{T}(B X-X B)=\operatorname{tr}(T(B X-X B))=0 .
$$

Hence $f_{T}$ annihilates $\mathscr{R}\left(\Delta_{B}\right)$ and we have $\Re\left(\Delta_{B}\right)^{-} \subset \mathcal{R}\left(\Delta_{A}\right)^{-}$.

For the opposite implication we reverse the above argument. To prove part (b) we replace $T \in \mathcal{F}$ by $T \in \mathcal{T}$.

A related question is if proper containment ever occurs between the weak* and weak closures of the range of a derivation. The following provides us with an example.

For $A \in \mathscr{B}(\mathcal{H})$, J. P. Williams [9] has shown that $\Re\left(\Delta_{A}\right)^{-}=\mathscr{B}(\mathcal{H})$ if and only if $\{A\}^{\prime} \cap \mathscr{F}=\{0\}$ and $\mathscr{R}\left(\Delta_{A}\right)=\mathscr{B}(\mathcal{H})$ if and only if $\{A\}^{\prime} \cap \mathcal{T}=$ $\{0\}$.

EXAMPLE. Let $V$ be the Volterra operator on $L^{2}[0,1]$ defined by $(V f)(x)=$ $\int_{0}^{x} f(t) d t$. The invariant subspaces of $V$ are the subspaces $L^{2}[a, 1]$ for $0 \leqslant a$ $\leqslant 1$ (see [4]). Therefore $\{V\}^{\prime} \cap \mathscr{F}=\{0\}$ and $\mathscr{R}\left(\Delta_{V}\right)^{-}=\mathscr{B}(\mathcal{H})$. Furthermore, $\{V\}^{\prime}$ does contain operators in $\mathcal{T}\left(V^{2}\right.$ for example), hence $\Re\left(\Delta_{V}\right)^{-} \neq$ $\mathscr{B}(\mathcal{H})$.

\section{REFERENCES}

1. J. Dixmier, Les fonctionnelles linéaires sur l'ensemble des opérateurs bornés d'un espace de Hilbert, Ann. of Math. 51 (1950), 387-408.

2. P. R. Halmos, A Hilbert space problem book, Van Nostrand, Princeton, N.J., 1967.

3. I. N. Herstein, Topics in algebra, Blaisdell, Waltham, Mass., 1964.

4. G. K. Kalisch, On similarity, reducing manifolds, and unitary equivalences of certain Volterra operators, Ann. of Math. 66 (1957), 481-494.

5. H. W. Kim, On compact operators in the weak closure of the range of a derivation, Proc. Amer. Math. Soc. 40 (1973), $482-486$.

6. D. C. Kleinecke, On operator commutators, Proc. Amer. Math. Soc. 8 (1957), 535-536.

7. R. Schatten, Norm ideals of completely continuous operators, 2nd printing, Ergebnisse der Mathematik und ihrer Grenzgebiete, Band 27, Springer-Verlag, Berlin, 1970.

8. R. E. Weber, The range of a derivation and ideals, Pacific J. Math. 50 (1974), 617-624.

9. J. P. Williams, On the range of a derivation, Pacific J. Math. 38 (1971), 273-279.

Department of Mathematics, Pennsylvania State University, Shenango Valley Campus, Sharon, Pennsylvania 16146 